\title{
Wireless Data Center with Millimeter Wave Network
}

\author{
Hars Vardhan, Navine Thomas, Seong-Ryong Ryu, Bhaskar Banerjee and Ravi Prakash \\ The Erik Jonsson School of Engineering \& Computer Science \\ The University of Texas at Dallas
}

\begin{abstract}
Data centers are becoming increasingly popular for a wide variety of applications such as search, gaming, email and so on. The complex wiring issues in data centers have motivated us to consider a wireless solution for data centers which meets the design goals of a typical data center network while providing the additional benefit of flexibility in the network. The wireless data center is based on the recently released IEEE $802.15 .3 \mathrm{c}$ wireless networking standard in the $60 \mathrm{GHz}$ ISM band. The standard supports multi-Gbps data rates and enables the transceivers to set up point-to-point links with each other, which makes it suitable for data center interconnects.

In this paper, we use $60 \mathrm{GHz}$ wireless technology to design a wireless data center and show how various data center interconnects such as 3-tier and fat-tree can be emulated on such a wireless network.
\end{abstract}

\section{INTRODUCTION}

A data center can be seen as a large interconnect of commodity computing and storage devices. To support the large scale of enterprise applications, the data center network (DCN) design requires careful consideration of performance, resilience, scalability as well as flexibility [1].

Several forms of proven layered DCN architectures (2-tier, 3 -tier) are being used by various vendors in the industry [1], [2], [3]. A two-tier design is used for a small number of hosts $(5-8 \mathrm{~K})$, while a 3-tier design is usually employed for larger data centers (around $25 \mathrm{~K}$ hosts). To address the scalability and performance issues of 2-tier and 3-tier designs, architectures such as fat-tree [4] and Dcell [5] have been proposed in the literature. However, some of the common issues of all these designs are complex wiring, cooling space and reconfigurability. The cost of cabling in a data center may be up to $7-8 \%$ of the infrastructure cost [6]. Also, the significant volume of space occupied by cables may degrade the overall cooling efficiency of data centers [7]. Furthermore, the wired topology causes network reconfiguration to be a difficult and tedious process. This is more critical in the case of modular data centers [8] (MDC) which are used for quick deployment and require high reconfigurability.

We propose to alleviate the issue of inflexibility in existing DCNs due to their fixed, wired topologies, without sacrificing scalability. Towards this goal, we describe a solution to emulate various DCN architectures with $60 \mathrm{GHz}$ millimeterwave wireless network. The recently released standard IEEE 802.15.3c [9] is capable of supporting multi-Gigabit data rate point-to-point links in the $60 \mathrm{GHz}$ ISM band. The small wavelength in the band enables placement of multiple antennas in close proximity, thus enabling beam-forming. We exploit beam-forming to create highly directional point-to-point links as described in Section III. Many such links can be concurrently setup in a given area. The increased atmospheric absorption and propagation loss in the $60 \mathrm{GHz}$ band limits the communication range $(<10 \mathrm{~m})$. However, this provides security over the wireless links and increases spatial reuse of the spectrum. In addition, we employ beam steering to switch the links from one node to another in order to achieve a highly reconfigurable DCN design. The high atmosphere absorption and propagation loss require that all communicating pairs be within line of sight (LOS) of each other. This challenge is successfully addressed in Section IV.

\section{BACKGROUND AND RELATED WORK}

A data center houses a large number of computational and storage resources interconnected with a set of routers and switches. The data center network must meet the following design goals: Scalability, Cost effectiveness, High throughput and Low oversubscription. Oversubscription is used to mean assigning a total bandwidth to a given network element that is greater than that element's capacity [4]. The approach is used to save money on the expensive network elements.

Another important feature of the DCN design is flexibility, i.e., the ability to support new applications and quickly deploy the system when implementing new services. Designing a flexible architecture with the ability to support entirely different data flow patterns has a significant advantage and can be easily achieved if we have on-the-fly reconfigurability as seen in wireless networks. Also, reconfigurability can provide graceful degradation in case of failures of components in the DCN.

Traditional DCN architectures are based on a proven layered approach. The layers of the architecture are core, aggregation, and access layers [1]. The core layer has redundant connectivity to multiple aggregation modules and is responsible for routing the flows going in and out of the data center. The aggregation layer provides module integration services between the core and access layers. The access layer is where the compute and storage servers are physically attached. The 2-tier architecture has core and access layers only whereas the 3-tier has all three layers.

3-tier Layered Architecture: There are many variations of the layered architecture (2-tier, 3-tier) which are deployed by leading vendors like Cisco, Sun and IBM. One module of a simple 3-tier layered architecture is shown in Fig. 1. It is 


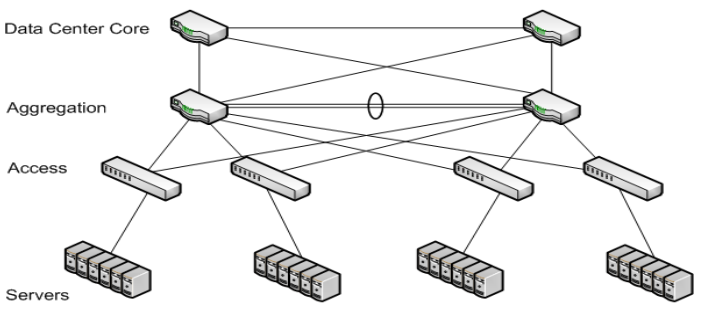

Fig. 1. 3- tier Layered Architecture

highly oversubscribed. High oversubscription adversely affects the performance and availability of the data center.

Fat-Tree Architecture: The traditional network architecture forms a tree of routing and switching elements with specialized and more expensive equipments moving up the interconnect layers. Unfortunately, even when deploying the highest-end IP switches/routers, resulting topologies may only support $50 \%$ of the aggregate bandwidth available at the edge of the network, while still incurring tremendous cost. So, a different hierarchical network structure has been proposed by Al-Fares et. al [4], in which commodity switches are used, and is proven to be more efficient and scalable. They adopted fat-tree [10] network for interconnect which is a special instance of the historically popular non-blocking Clos topology [11]. A simple fat-tree topology is shown in Fig. 2. The fat-tree architecture

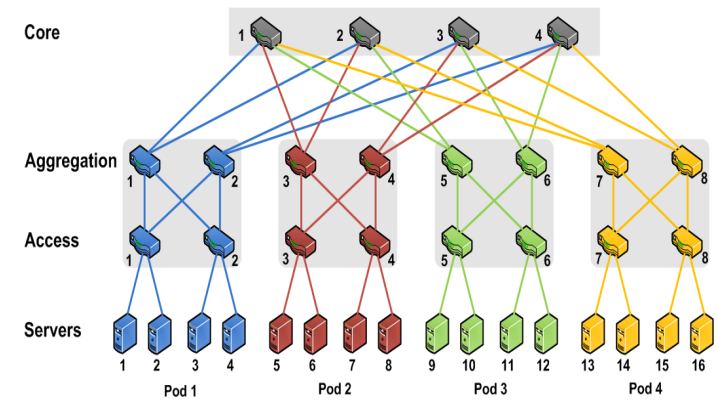

Fig. 2. Fat Tree Architecture

is very promising in terms of scalability, cost and simplicity of the interconnection.

Both the 3-tier and the fat-tree architectures could be implemented using $60 \mathrm{GHz}$ wireless links, with the added benefits of increased reconfigurability and flexibility. For example, adding a few servers to the DCN may require a set of links to be reconnected. For wired DCNs, this can be a time-consuming and error prone process. Whereas, with beam-forming and beam-steering, the same can be accomplished and verified almost instantaneously using $60 \mathrm{GHz}$ links.

A more scalable DCN architecture, namely DCell, has been proposed in [5]. Also, a specifically designed network architecture (BCube) for Modular data center is described in [12]. DCell and BCube both employ a recursive approach and use mini switches for interconnections. While $60 \mathrm{GHz}$ wireless links can be used to interconnect DCell and BCube, we omit the details due to space limitation.
Ramachandran et al. [13] wrote one of the earliest papers advocating the use of $60 \mathrm{GHz}$ wireless links to replace some of the wired links in a DCN with the primary aim of reducing wiring clutter. They proposed the use of LOS links where necessary and NLOS links using reflectors. However, they did not describe ways to emulate specific DCN topologies like fat-tree and they mentioned beam-forming and antenna patterns as open problems. Our work extends theirs as we describe how specific DCN topologies can be realized using only steerable (using an array of antennas) point-to-point (with beam forming) wireless links in the $60 \mathrm{GHz}$ band. This creates the possibility of a completely wireless DCN.

\section{Millimeter wave and Transceiver Design}

The recently proposed IEEE802.15.3c standard [9], also known as $60 \mathrm{GHz}$ millimeter-wave (mmWave) MAC/PHY, was developed for WPANs (Wireless Personal Area Networks). However, it can be applied to wireless DCNs because of the variety of capabilities it offers such as high data rates, ad hoc connectivity, support for beam forming, reliability and security. The mmWave PHY operating frequency is within the $57.0-66.0 \mathrm{GHz}$ range as allocated by the regulatory agencies in Europe, Japan, Canada and the United States. Four channels have been proposed over this ISM band and each of them has approximate bandwidth of $2.16 \mathrm{Gbps}$. The standard specifies three PHY modes - Single Carrier (SC PHY), High Speed Interface (HSI PHY) and Audio/Visual (AV PHY) - to cater to the different market segments. For the mmWave wireless DCN, we propose to employ SC PHY due to its low power requirements. Though HSI PHY and AV PHY modes have freedom of having non LOS links, they may not be suitable for dense communication networks.

The standard describes a piconet as a wireless ad hoc data communications system which allows a number of independent data devices (DEVs) to communicate with each other on a selected channel [9]. It supports directional peer communication, i.e., the DEVs in the piconet can communicate with a piconet network controller (PNC) and also with each other using a beam forming protocol. Directional communication has advantages, such as low interference, point to point high speed link and spatial re-use of spectrum. These unique characteristics make the $60 \mathrm{GHz}$ network significantly different from the traditional $2.4 \mathrm{GHz}$ and $5.8 \mathrm{GHz}$ licensefree radio networks. The standard uses various modulation and coding schemes to enhance the throughput even further.

Transceiver design for $60 \mathrm{GHz}$ network: The wireless communication system operated at $60 \mathrm{GHz}$ frequency exhibits several design challenges due to losses such as atmospheric absorption loss and propagation loss. The absorbed electromagnetic (EM) energy around $60 \mathrm{GHz}$ by Oxygen is typically $15-30 \mathrm{~dB} / \mathrm{km}$. Free space propagation loss resulting from the reduced power density through free space, might be the most significant loss in moderate distance communication and it is inversely proportional to $(l f)^{2}$, where $l$ is the distance between a pair of transceivers and $f$ is the carrier frequency, respectively [14]. The Oxygen absorption and propagation loss of the 
$60 \mathrm{GHz}$ signals limit the coverage distance $\left(D_{\max }<10 \mathrm{~m}\right)$.

Beam-forming with phased-array can be employed in the transceiver in order to minimize the propagation loss and increase the directivity. Figs. 3(a) and 3(b) show the radiation patterns of single isotropic and phased-array antennas respec-

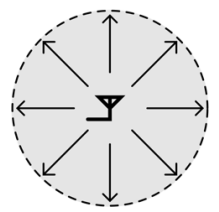

(a) Single isotropic antenna

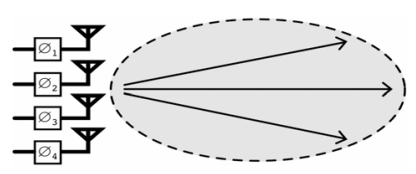

(b) Phased-array
Fig. 3. Radiation Patterns

tively. As seen, the single isotropic antenna radiates EM waves in all direction, while the narrower beam-shaped radiation pattern is formed with multiple antennas. In fact, as more antennas are used, the beam width obtained becomes narrower and prevents the EM radiation energy from spreading out in all directions. As a result, the propagation loss is further reduced and a point-to-point link can be formed. Another advantage of phased-array is that interference and multi-path effect can be also minimized due to its narrow beam width and high directivity [15].

The required beam width can be directly determined by both the minimum inter-distance $(X)$ between two adjacent transceivers and the longest distance $\left(D_{\max }\right)$ of mmWave link. The Beam width $\left(\theta_{W}\right)$, in the case of isotropic antennas, is defined as $\frac{1}{(m-1)}$ radian, where $m$ is the number of antennas. Therefore, a relation can be derived between parameters $X$, $D_{\max }$ and $m$ as given in eq. 1 , where $X$ is assumed to be much greater than $D_{\max }$.

$$
\theta_{W}=\frac{360^{\circ}}{(m-1) \pi}=\arctan \left(\frac{X}{D_{\max }}\right) \approx \frac{X}{D_{\max }}
$$

It can also be noted that the required antenna distance of the array is $\frac{\lambda}{2}$, where $\lambda$ is the wavelength and is only $5 \mathrm{~mm}$ at 60 GHz. With such a high frequency, the physical dimension of the antenna shrinks and can be as small as a US dime [16]. Therefore, it is feasible to build sufficient number of antennas on a small area to make $\theta_{W}$ as small as $5^{\circ}$.

In order to achieve point-to-point communication between servers with the phased-array, beam steering (by controlling beam angles) is also required and it can be accomplished by using phase rotators as shown in Fig. 3(b). Each phase rotator changes the signal phase $\left(\theta_{R}\right)$ in different antenna elements of the array and thus controls the beam direction in which EM energy is transmitted by the antenna array [17].

For the functional verification, the phased rotator is designed using IBM 90-nanometer CMOS process and its part of schematic is shown in Fig. 4(a) [18]. The phase rotator can provide the signal phase shift by using amplitude difference of in-phase signals $\left(V_{I+,-}\right)$ and quadratic-phase signals $\left(V_{Q+,-}\right)$, e.g. $A_{1} \cos (\omega t)+A_{2} \sin (\omega t)=A_{3} \sin (\omega t+\theta)$ where $\theta$ is $\tan ^{-1} \frac{A_{1}}{A_{2}}$. Fig. 4(b) shows the simulation results of the phase rotation from $0^{\circ}$ to $180^{\circ}$ with increment of $11.25^{\circ}$ (5.2 picosecond). Lastly, Fig. 4(c) shows $\approx 300$ nanosecond of the simulated transient response, which means that DCN links can be broken/established in 300 nanosecond enabling reconfiguration of the interconnect on the fly. Though the maximum beam steering angle $(\theta)$ achieved is $180^{\circ}$, however, it performs best in the range of $140^{\circ}-160^{\circ}$ in our arrangement.

$D_{\max }$ together with $\theta$ defines a conical region within which a transceiver can steer the beam to make point-topoint links with other transceivers in the region. However, the beam steering can be done only in steps of $\theta_{R}$, i.e., a transceiver $A$ can switch the beam from node $B$ to node $C$ if $\angle B A C=n . \theta_{R}, n \in I^{+}$. The angle $\angle B A C=\alpha$ (Fig. 7(b)) can be computed using geometric formula given in eq. 2 .

$$
\alpha=\arctan \left(\frac{h 2}{y}\right)-\arctan \left(\frac{h 1}{y}\right)
$$

It can be seen that the smaller the value of the phase rotation step $\left(\theta_{R}\right)$, the greater the number of potential simultaneous point-to-point links within the conical region $(\theta)$.

\section{Wireless Data Center Network}

To replace the wired (Ethernet) links with wireless links, we first compare the relevant parameters of both Ethernet [19] and IEEE802.15.3c [9] wireless links as shown in Table I. Most of

\begin{tabular}{|l|c|c|}
\hline Feature & Ethernet & IEEE 802.15.3c \\
\hline Link speed & $2-10 \mathrm{Gbps}$ & $2.5-5.7 \mathrm{Gbps}$ \\
\hline Bit error rate & $10^{-13}$ & $10^{-12}$ \\
\hline Comm. Range & $400-2000 \mathrm{~m}$ & $10 \mathrm{~m}$ \\
\hline \multicolumn{3}{|c|}{ TABLE I } \\
ETHERNET VS MMWAVE
\end{tabular}

the IEEE803.15.3c parameters are as good as that of Ethernet technology. The short communication range of mmWave is not an issue in data center applications because of the close proximity of servers and switches which need to communicate with one another. Hence, use of $60 \mathrm{GHz}$ mmWave links in data centers is viable. Wireless DCN can provide additional features to the data center networks, such as reduced complex wiring cost, decreased cooling cost due to reduced wiring clutter and reconfigurability.

\section{A. Hexagonal Arrangement}

Typically, the server racks in a data center are arranged in rows with corridors between them. A rack has many slots, also called rack units (RU), to contain nodes (servers/network equipments). The standard width of the rack $\left(W_{\text {rack }}\right)$ is $50 \mathrm{~cm}$ and the height of each RU $\left(H_{R U}\right)$ of the rack is $4.25 \mathrm{~cm}$. The standard rack height $H_{\text {rack }}$ is 40 times $H_{R U}$, i.e, 40 nodes can be placed in a rack. The logic behind the row-wise arrangement is to allow easy access to the front and rear of the servers. However in order to use the $60 \mathrm{GHz}$ technology effectively, the nodes would have to be arranged such that the ends with the transceivers (integrated on RUs) face each 


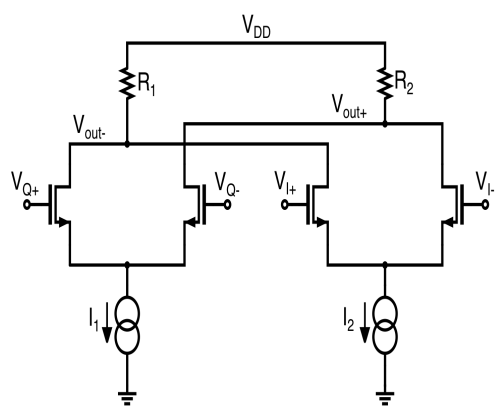

(a) Schematic of a phase rotator cell

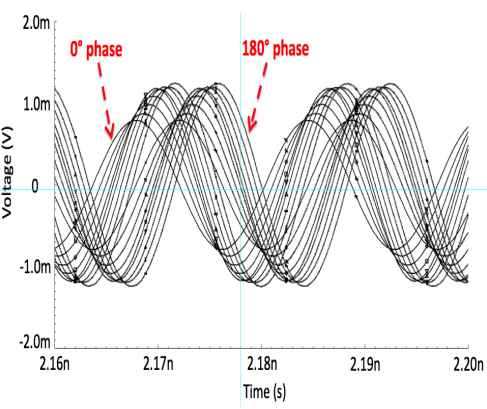

(b) Phase rotation simulation

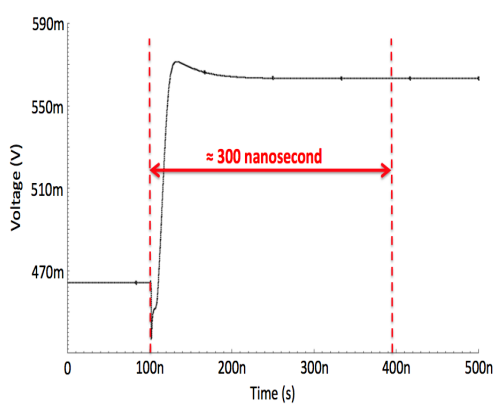

(c) Transient response simulation

Fig. 4. Schematic and simulation results

other so that their antennas can establish the LOS wireless channels. This implies that the servers in a rack would not be able to communicate with each other and further if racks are placed side by side to form a row, then all the servers within a row would not be able to communicate directly with one another. In such a scenario, the possible ways to arrange the nodes are to either place the server racks in two parallel rows with the transceivers facing each other or to place the racks on the edges of an n-sided regular polygon such as a triangle, square or hexagon. When the racks are placed along edges of a regular polygon, the space in which LOS wireless links are formed is enclosed. This restricts obstructions to communication caused by human movements between the racks. Our aim is to have a uniform arrangement where a node can communicate with a sufficient number of other nodes. The number of potential nodes to communicate with for a transceiver is directly proportional to the rack area contained in the conical region within which a transceiver can steer its beam as explained in Section III. However, when comparing the different rack arrangements, we only consider the number of accessible nodes on the same horizontal plane (RUs on same height from bottom) since this can then be easily extended to the vertical plane. So, in the following texts, an analysis of the fraction of accessible nodes for a source transceiver in a horizontal slice of the different arrangements is provided.

Two important parameters that determine the number of potential communicating nodes for a source transceiver are - the maximum distance for communication in the horizontal plane, $D$ and the maximum phase rotation angle, $\theta$. First, the arrangement of servers on two parallel rows, each of length $L$, is shown in Fig 5(a). A server on Row 2 can communicate

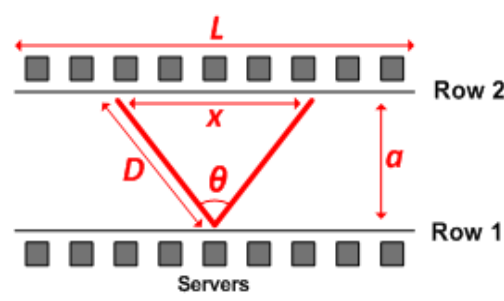

(a) Two parallel rows

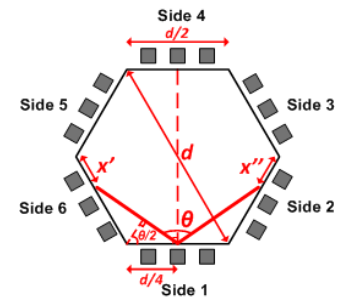

(b) Hexagon shape
Fig. 5. Nodes arrangement with nodes along length $x$ of Row 1 , where $x=2 D \sin \frac{\theta}{2}$. Let $P$ be the total length of the two rows and $\mathrm{N}$ be the total number of servers over $P$. Then the fraction of the total number of servers, a server in the center of a row can communicate with is given as $R=\frac{x}{P} N$. In the ideal case, $L=x=\frac{P}{2}$ and $R=0.5 N$, i.e, a node on the center of a row can communicate with half of the total number of servers on the two rows. However, as we move away from the center, the number of servers that a node can communicate with decreases proportionally. Therefore, the average fraction of servers that any server in a row can communicate with would be $R=\frac{1}{2 x} \frac{x+\frac{x}{2}}{2} N \Longrightarrow R=0.375 N$.

Next, consider the arrangement of servers on the edges of a regular hexagon as shown in Fig. 5(b). The constraint in such an arrangement is that in order for all nodes to be in communication range of one another, the maximal diameter of the hexagon, $d \leq D$ For $\theta>100$, a server on the center of side 1 can communicate with all the servers on sides 3,4 and 5 and also with servers along $x^{\prime}$ and $x^{\prime \prime}$ on sides 2 and 6 respectively. For a server at the center of side $1, x^{\prime}$ and $x^{\prime \prime}$ are computed in equation 3

$$
x^{\prime}=x^{\prime \prime}=\frac{d}{2} \frac{\sqrt{3} \sin \theta / 2-2 \cos \theta / 2}{\sqrt{3} \sin \theta / 2-\cos \theta / 2}
$$

Therefore, the total length, $x=3 a+2 x^{\prime}$. If $P^{\prime}$ is the perimeter of the hexagon and $N^{\prime}$ the total number of servers over $P^{\prime}$, then the fraction of the total number of servers a server in the center of a side can communicate with is given in eq. 4 .

$$
R=\left(x / P^{\prime}\right) N^{\prime}=\frac{1}{6} \frac{5 \sqrt{3} \sin \theta / 2-7 \cos \theta / 2}{\sqrt{3} \sin \theta / 2-\cos \theta / 2} N^{\prime}
$$

It can be shown that this fraction holds good for not just the node at the center of a side but to any node on the side. In order to compare the hexagon with the two-row arrangement, it is concluded that for $\theta>100$, the hexagon constructed will have $P^{\prime}<P$, for $d=D$. Therefore, the number of nodes in the hexagon is $N^{\prime}=\frac{P^{\prime}}{P} N$. Substituting this in eq. 4, the hexagon and two-row arrangements can be compared for different phase rotation angles $(\theta)$. The fraction, $R$, is derived in a similar manner for other polygons.

Fig. 6 shows the values of fraction $R$, varying $\theta$ from 
$100^{\circ}$ to $160^{\circ}$ for various arrangements. It can be seen that the fraction of nodes that a node can access is greater in the hexagon than those in the other arrangements for the desired angles. The octagon performs close to the hexagon however those are not high enough to justify the increased complexity in such an arrangement. Therefore, we prefer a hexagonal arrangement for nodes in this paper, i.e., the server rack would be placed on the edges of a regular hexagon to form a hexagonal prism structure.

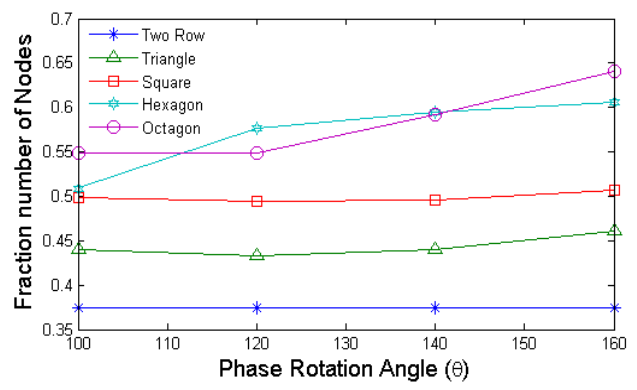

Fig. 6. Fraction of nodes accessible at different phase rotation angles

\section{B. Emulating DCN with Wireless Links}

We propose the idea of having two transceivers on each node mounted on an RU, such that traffic passing through the node can be handled without frequent beam switching of high data rate links from one communicating node to another. Additionally, it provides four options for LOS connectivity between a pair of nodes and this increases the flexibility of the wireless DCN. For simplicity of analysis, hereafter, it is assumed that there is only one rack on each side of the hexagon.

Considering the hexagon arrangement with each side of length $L$ and two transceivers per node on each RU of a side, it can be shown that, in a horizontal plane, a node can steer the beam of at least one of its transceivers to five other nodes. This is shown in Fig. 7(a) assuming $\theta=120$. In Fig. 7(a), 12 transceivers (two on each side) are placed on the sides with an angular distance of $\frac{360^{\circ}}{12}=30^{\circ}$ between adjacent transceivers and at equal distances from the center of the plane. The transceiver $T_{0}$ can steer the beam (by $3 \theta_{R}=30^{\circ}$ ) to establish the LOS links with $T_{1}, T_{3}, T_{5}, T_{7}$ and $T_{9}$. Similarly, $T_{11}$ can communicate with the transceivers $T_{2}, T_{4}, T_{6}, T_{8}$ and $T_{10}$.

Also, the nodes can steer their beams vertically to communicate with other nodes in LOS subject to the limitation imposed by the maximum beam steering angle $(\theta)$ and communication range $\left(D_{\max }\right)$, i.e., the conical region described in Section III. For all nodes of the hexagonal prism to be within the communication ranges of each other, $L<\frac{1}{2} \sqrt{D_{\max }^{2}-\left(H_{\text {rack }}\right)^{2}}$, which holds true for $D_{\max }=10 \mathrm{~m}, H_{\text {rack }}=170 \mathrm{~cm}$ and $L=50 \mathrm{~cm}$.

Furthermore, the number of nodes to which a node can steer its beam in the vertical plane of the hexagonal prism can be determined by computing the vertical distance enclosed in the conical region as shown in Fig. 7(b). For the hexagonal

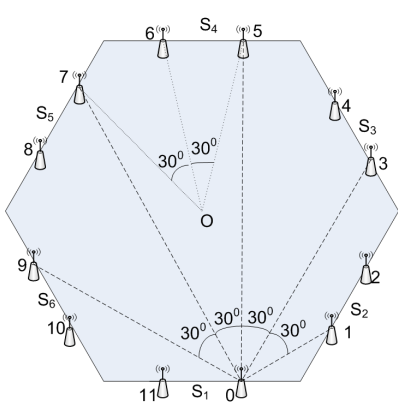

(a) Horizontal Plane

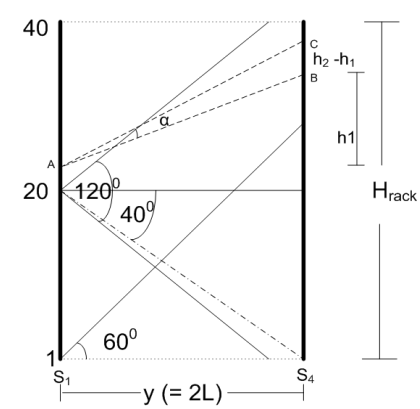

(b) Vertical Plane
Fig. 7. Phase rotation and angular separation

arrangement, the distance between $S_{1}$ and $S_{4}$ is $2 L$. The conical region (for $\theta=120^{\circ}$ ) with the apex at the node 20 of $S_{1}$ encloses all nodes of $S_{4}$, and the angle made on the node 20 by $S_{4}$ can be computed as $80^{\circ}$. Hence, node 20 can communicate with $\frac{80}{\theta_{R}}+1=9$ nodes on $S_{4}$. On the other hand, only half of the conical region $\left(\frac{\theta}{2}=60^{\circ}\right)$ with apex at node 1 (or 40) encloses the vertical area of $S_{4}$. So, it can be shown that the number of nodes accessible by node 1 (or 40) is $\frac{60}{\theta_{R}}+1=7$. Therefore, the average number of accessible nodes on the vertical face of $S_{4}$ by a node on $S_{1}$ turns out to be 8 out of 40 , which is $\frac{1}{5}^{\text {th }}$ of the total RUs in a rack. Similarly, a node can communicate with $\frac{1}{5}^{\text {th }}$ of the RUs placed on any of the other sides $S_{2}, S_{3}, S_{5}$ or $S_{6}$. Effectively, a given node may be able to communicate with $\frac{1}{6}\left(\frac{1}{5}+2 \frac{1}{5}+2 \frac{1}{5}\right)=\frac{1}{6}$ of the total RUs in the DCN, if there is only one piconet in the system. Upon adding another piconet on an orthogonal channel we can double this fraction to $\frac{1}{3}$, which should suffice for most of the DCN architectures. The rest of the section describes how different data center architectures can be realized with only mmWave wireless LOS links. We assume that all servers are identical and switches of the layers are identical.

a) Emulating 3-tier Architecture: The 3-tier layered DCN architecture shown in Fig. 1 can be emulated using only LOS links. The layered architecture resembles a bipartite graph, which can be partitioned into two or more independent sets of nodes such that no two nodes within a set need to communicate with each other. For example, in case of a single rack on each side of the hexagonal arrangement which can thereby accommodate a total of 240 RUs, a layered DCN architecture can have one core router (4 RUs), 8 aggregation routers, 32 access routers and 128 servers. The core routers need not to communicate with the access routers and the aggregation routers need not to communicate with the servers. This configuration can be emulated by applying Algorithm 1 while assigning nodes to the racks.

b) Emulating Fat Tree: A fat-tree with $k$-port switches has $k$ pods. Each pod has $k$ switches, half of which are in the access layer while the other half of them are in the aggregation layer. Using $k$-port switches, a fat-tree interconnect can have $\frac{k^{3}}{4}$ number of hosts and requires a total of $\frac{5 k^{2}}{4}$ number of switches [4]. Each node in a $k$-pod fat-tree needs to 


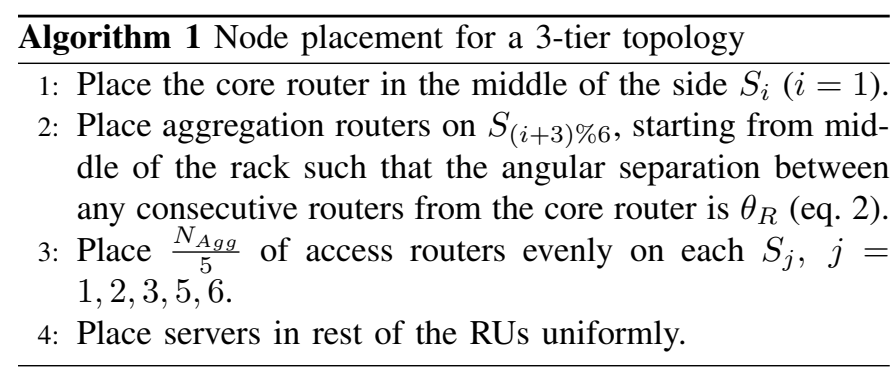

communicate with $k$ other nodes among $\frac{5 k^{2}+k^{3}}{4}$ nodes. The fraction $k /\left(\frac{5 k^{2}+k^{3}}{4}\right)$ is $\leq \frac{1}{3}$ for $k \geq 3$, so each node has a sufficient number of communication links if we use two piconets on two orthogonal channels. The graph induced by the fat-tree network topology is also a bipartite graph, therefore the topology can be emulated in a hexagonal arrangement. For example, the fat-tree topology shown in Fig. 2 can be emulated with only LOS links employing Algorithm 2 as shown in Fig. 8 .

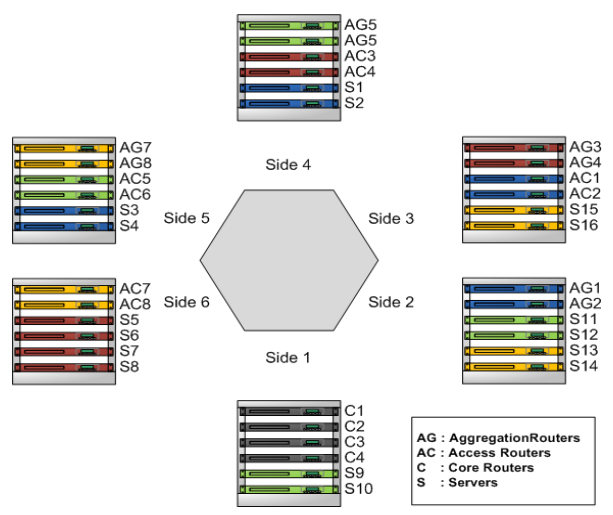

Fig. 8. Fat Tree with LOS links

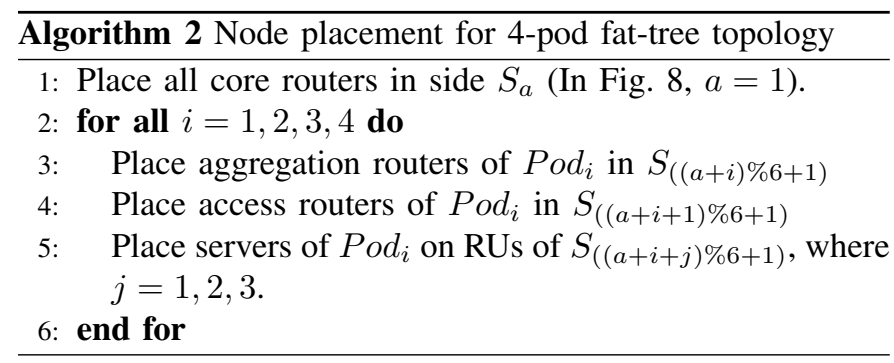

Having two transceivers on each RU gives more flexibility in creating two piconets on orthogonal channels and hence concurrent transmissions can be maximized in the wireless DCN. The core router(s) and one of the transceivers of each aggregation router can make one piconet, while the remaining transceivers of the aggregation routers and the other remaining nodes can form another piconet. Further, we can even create a third piconet in a similar way for larger DCNs. Thus, it is possible to emulate a DCN with IEEE 802.15.3c network which is flexible due to a sufficient number of nodes being within the line of sight of each other.

\section{CONClusion}

The wired connectivity in a DCN suffers from issues like inflexibility and wiring clutter. To address these, we have studied the $60 \mathrm{GHz}$ wireless network and proposed the design of a transceiver which provides point-to-point LOS links using beam-forming and beam steering. Ethernet comparable high data rate and point-to-point wireless links in the $60 \mathrm{GHz}$ band make it suitable for data centers. We proposed methods for placement of nodes to emulate 3-tier and fat-tree data center interconnects with such wireless links. This creates the possibility of having a completely wireless DCN.

\section{REFERENCES}

[1] "Cisco Data Center Infrastructure 2.5 Design Guide," Cisco Systems, Inc, December 2007. [Online]. Available: http://www.cisco.com

[2] "Government Data Center Network Reference Architecture," Juniper Networks, Inc., 2009. [Online]. Available: http://www.juniper.net/

[3] "Data Center Solutions Overview," Extreme Networks, Inc., 2009. [Online]. Available: http://www.extremenetworks.com/

[4] M. Al-Fares, A. Loukissas, and A. Vahdat, "A scalable, commodity data center network architecture," in SIGCOMM '08: Proceedings of the ACM SIGCOMM 2008 conference on Data communication. New York, NY, USA: ACM, 2008, pp. 63-74.

[5] C. Guo, H. Wu, K. Tan, L. Shi, Y. Zhang, and S. Lu, "Dcell: a scalable and fault-tolerant network structure for data centers," SIGCOMM Comput. Commun. Rev., vol. 38, no. 4, pp. 75-86, 2008.

[6] (2006, October) Structured Cabling: India on a live wire. Voice and Data. [Online]. Available: http://tinyurl.com/5nxe2m

[7] Optimized airflow assessment for cabling. IBM IT Services. [Online]. Available: http://tinyurl.com/69u957

[8] "Containerized Datacenters," Sun Mircosystems, Inc., February 2009. [Online]. Available: http://www.sun.com/service/sunmd/

[9] 802.15.3c: IEEE Standard for Information Technology - Telecommunications and Information Exchange Between Systems - Local and Metropolitan Area Networks, IEEE Std., 2009.

[10] C. E. Leiserson, "Fat-trees: universal networks for hardware-efficient supercomputing," IEEE Trans. Comput., vol. 34, no. 10, pp. 892-901, 1985.

[11] C. Clos, "A study of non-blocking switching networks," Bell System Technical Journal, Tech. Rep. 32(2), 1953.

[12] C. Guo, G. Lu, D. Li, H. Wu, X. Zhang, Y. Shi, C. Tian, Y. Zhang, and S. Lu, "BCube: a high performance, server-centric network architecture for modular data centers," in SIGCOMM '09: Proceedings of the ACM SIGCOMM 2009 conference on Data communication. New York, NY, USA: ACM, 2009, pp. 63-74.

[13] R. M. K. Ramachandran, R. Kokku and S. Rangarajan, "60 $\mathrm{GHz}$ Data-Center Networking: Wireless $\Longrightarrow$ Worry less?" NEC Laboratories America, Inc., Tech. Rep., July 2008. [Online]. Available: http://www.nec-labs.com/ kishore/papers/60GHz-DC.pdf

[14] C. Doan, S. Emami, D. Sobel, A. Niknejad, and R. Brodersen, "Design considerations for $60 \mathrm{GHz}$ CMOS radios," Communications Magazine, IEEE, vol. 42, no. 12, pp. 132 - 140, dec. 2004.

[15] K.-C. Huang and D. J. Edwards, Millimetre Wave Antennas for Gigabit Wireless Communications: A Practical Guide to Design and Analysis in a System Context. Wiley Publishing, 2008.

[16] B. Floyd, S. Reynolds, U. Pfeiffer, T. Beukema, J. Grzyb, and C. Haymes, "A silicon $60 \mathrm{GHz}$ receiver and transmitter chipset for broadband communications," in Solid-State Circuits Conference, 2006. ISSCC 2006. Digest of Technical Papers. IEEE International, Feb. 2006, pp. 649-658.

[17] K.-J. Koh, J. May, and G. Rebeiz, "A millimeter wave $(40-45 \mathrm{GHz})$ 16-element phased-array transmitter in $0.18-\mu \mathrm{m} \mathrm{SiGe} \mathrm{BiCMOS}$ technology," in IEEE J. SSC, Vol. 44, No. 5, May 2009.

[18] K.-J. Koh and G. Rebeiz, "0.13- $\mu \mathrm{m}$ CMOS Phase Shifters for X-, Ku, and K-Band Phased Arrays," Solid-State Circuits, IEEE Journal of, vol. 42, no. 11, pp. $2535-2546$, nov. 2007.

[19] IBM ethernet switch B24X specification. IBM IT Services. [Online]. Available: http://tinyurl.com/ykydt9k 